\title{
Improved Technology for Hard Anodizing Dissolution of Aluminum Alloy Part
}

\author{
Lixia Peng ${ }^{*}$ and Man Li \\ Department of Automation Engineering, Xi'an International University, Xian, Shanxi, 710077, China
}

\begin{abstract}
This paper analyzes the main factors in the process of dissolution of hard anodized aluminum alloy, together with the specific problems in the production practice, introduces in detail the methods of analysis, finds out the causes of dissolution, and puts forward the pertinent improvement measures. This paper is of instructive significance to effectively resolve the problems of dissolution in the process of tube parts hard anodizing.
\end{abstract}

Keywords: Aluminum alloy, dissolution, hard anodizing.

\section{INTRODUCTION}

Aluminum alloy is the optimum lightweight material the aerospace industry. The hardness, anti-corrosion, insulation, resistance to wear, and high temperature resistance of which are all increased significantly after processed by hard anodizing, and therefore it has been gradually receiving wide attention due to increased applications. Though the application of technology of hard anodizing of aluminum alloys is wide [1], and it has been a rather mature surface treatment process, during the course of oxidation, influenced by the quality of oxidation film, there will inevitably occur various problems, among which the partial or complete dissolution of the parts is one of the most common phenomena that affect the quality of surface treatment of parts $[2,3]$. In this paper, various methods have been put forward for analyzing the problems of hard anodizing dissolution of aluminum alloy part. Taking the parts in real production, the problem of matrix dissolution is deeply discussed, and some constructive improved technology has been put forward.

\section{THE MECHANISM OF HARD ANODIZING OF ALUMINUM ALLOY PART}

The growth of anode oxide film is conducted in a conflicting process of the thickening and dissolution of film [4]. When the electrolyte is energized, a layer of $\mathrm{AL}_{2} \mathrm{O}_{3}$ film will be formed on aluminum substrate surface, which chemical reaction is as follows:

$$
\begin{aligned}
& 2 \mathrm{OH}^{-}-2 e \rightarrow \mathrm{H}_{2} \mathrm{O}+\mathrm{O}^{2-} \\
& 2 \mathrm{AL}^{3+}+3 \mathrm{O}^{2-} \rightarrow \mathrm{AL}_{2} \mathrm{O}_{3}+Q \\
& 6 \mathrm{OH}^{-}+2 \mathrm{AL}^{3+} \rightarrow 3 \mathrm{H}_{2} \mathrm{O}+\mathrm{AL}_{2} \mathrm{O}_{3}+Q
\end{aligned}
$$

Meanwhile, the electrolyte can dissolve the aluminum and the oxidation film, which chemical reaction is as follows:

$$
\begin{aligned}
& 2 \mathrm{AL}+6 \mathrm{H}^{+} \rightarrow 2 \mathrm{AL}^{3+}+3 \mathrm{H}_{2} \\
& \mathrm{AL}_{2} \mathrm{O}_{3}+6 \mathrm{H}^{+} \rightarrow 2 \mathrm{AL}^{3+}+3 \mathrm{H}_{2} \mathrm{O}
\end{aligned}
$$

The growth process of oxidation film is shown in Fig. (1). Because of the strong appetency of oxygen and aluminum, the surface of aluminum will swiftly develop a layer of compact nonporous oxidation film the moment the electrolyte is energized, and the thickness varies from 10 to $50 \mathrm{~nm}$ based on the cell voltage. The oxidation film is highly insulative, which is called barrier layer [5]. Since the volume will expand when aluminum oxide is formed, the barrier layer becomes rough and uneven in surface. First of all, the oxidation film is dissolved by electrolyte and holes will emerge at the thin film layer. Then, the electrolyte enters into the surface of aluminum matrix through the holes, and the electrochemical reaction will continue, which makes the cavity changed into porous layer of dozens $\mu \mathrm{m}$. As the electrolysis goes on, the porosity of the porous layer will become deeper and deeper, and barrier layer begins to develop in the direction of aluminum matrix gradually. Finally, the porous oxidation film that is compounded by barrier layer and porous layer is formed. The development process of film is a synergistic effect between electrochemical reactions and chemical reactions. When the electrochemical reactions are quicker than the chemical ones, the film will have a net growth and its thickness is increased; on the contrary, oxidation film will be dissolved and the thickness will decrease. Given enough time, the formation of oxidation film will be completely dissolved by the electrolyte [6-8].

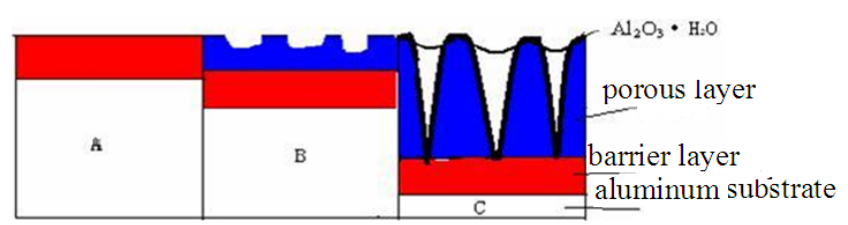

Fig. (1). Growth process of hard anodizing film. 


\section{THE REASON FOR ANALYSIS AND CONTROL MEASURES OF HARD ANODIZING DISSOLUTION OF ALUMINUM ALLOY PARTS}

The thickness and hardness of hard anodizing film is closely related to the concentration, temperature of electrolyte, current density, oxidation time, alloy composition and other factors [9]. Also, the dissolution of the parts is mainly determined by the factors listed above.

\subsection{The Influence of Electrolyte Concentration}

Generally speaking, in the craft of sulfuric acid hard anodizing, if the content of sulfuric acid is low, the hardness of oxidation film will be high. But for the aluminum alloy which is of high copper content, it is not advisable to adopt the low concentration of electrolyte; otherwise, it will cause parts dissolving. Thus, electrolyte should be selected according to the material. In mixed acid hard anodizing craft, organic acids with certain components should be added to raise the oxidizing temperature and reduce production difficulty [10].

\subsection{The Influence of Temperature of Electrolyte}

In general, in the stipulated range, the hardness and abrasive resistance of the film will be gradually improved as the temperature is reduced. In order to get oxidation film of high quality, in the oxidation process the temperature should be fluctuated as lightly as possible, which should be under the control of $\pm 2^{\circ} \mathrm{C}$. The excessive electric current will cause the failure of partial heat dissipation, even complete or partial dissolution of oxidation film. Therefore, in order to make the temperature of electrolyte at its best range, aluminum anodizing often needs to be equipped in the bending cooling pipe or other cooling equipment in the wall of electrolyzer.

\subsection{The Influence of Current Density}

The enlargement of current density can improve the growth rate of oxidation film and cut down the time required by the thick film. However, when the current density reaches its maximum limit, it will get the verse result, as the excessively high current density will enlarge the heat effect in porosity of oxidation film, which accelerates the dissolution of oxidation film. Of course, the excessively low current density cannot be allowed, which will widely reduce the growth speed of oxidation film. Practice shows that it is better to control current density at the range of $(0.8 \sim 2.5)$ $\mathrm{A} / \mathrm{dm}^{2}$. In the process of oxidation, the smaller the fluctuation of current density, the better the effect will be. At the initial stage of oxidation, the production operation should be implemented by the way of gradually increasing current density to the specified figure within a certain period of time $(0.5 \sim 2(\min ))$ [11].

\subsection{The Influence of Oxidation Time}

Oxidation time should be chosen according to the composition of electrolyte concentration and temperature, current density and thickness of oxide film [12]. In the same cell liquid, if the temperature of electrolyte is lower, and the current density is higher, the growth speed will be quicker, and the oxidation time to get the regulated thickness of oxide film will be shorter, and vice versa. At the initial stage, the development of thickness of oxidation film is almost in a straight line; however, as the time of oxidation extends, the thickening process of the oxidation film will gradually slow down, and finally, it will stop. In general, the oxidation time will not be over $60 \mathrm{~min}$.

\subsection{The Influence of Alloy Composition}

The aluminumalloy of different components of organization is largely different in the appearance of the oxidation film. The purer the aluminum alloy is, the easier it will be to get colorless, transparent and shining decorative oxidation film; whereas the higher the content of alloy is, the lower the transparent and shining degree and the worse the corrosion resistance of oxidation film will be. If the content of copper or silicon of aluminum alloy is higher, anodic oxidation becomes more difficult [13]. Because of the loose organization, various micro hole defects and uneven organization and other factors of cast aluminum alloy, and anodic oxidation should be specially taken care of, or else, it will be easy to be dissolved.

\subsection{The Main Measurement of Avoiding Dissolution}

In order to effectively avoid dissolution in anodizing, we can take the following measurements: (1) to select proper power and appropriate current density and current waveform; (2) to adopt effective measures to quickly dissipate the heat over the surface of the oxidizing objects; (3) to choose different additives and current waveform according to different composition of the alloy; (4) to ensure that the contact between artifact and tooling is well and the cathode conductivity is well too; (5) to avoid the mixture of different batches in the process of the hard anodizing of different aluminum alloy parts, as the influence of hard anodizing on alloy is greater than conventional hard anodizing.

\section{THE CASE ANALYSIS OF THE PROBLEM OF HARD ANODIZING DISSOLUTION OF ALUM- INUM ALLOY PARTS}

The material of some aviation bushing parts is LD10, the whole part is in the shape of a pipe, and its length is $174 \mathrm{~mm}$ and inner diameter is $\Phi 25.5 \mathrm{~mm}$, the maximum of outside diameter is $\Phi 30 \mathrm{~mm}$, the thickness of wall of parts is $2.25 \mathrm{~mm}$, and the thinnest part is $2 \mathrm{~mm}$. In the process of production, the middle sulfuric acid solution is used to adopt constant-voltage method for hard anodizing. The highest pressure of film growth process is $60-70 \mathrm{v}$; the bath temperature is controlled around $-5^{\circ} \mathrm{C}$. The parts are hung in a vertical way. The bottom of hard anodized part should be arranged to let the compressed air pass or mix. In hard anodizing process of a group of 49 parts, 24 of which are dissolved, and the dissolution mainly occurred in the lower or middle parts.

\subsection{The Analysis of Causes of Material and Technological Parameter}

For this component, since LD10 material is also selected among other products, and the routine is fixed, 
technological parameter and voltage curve applied is provided automatically by the equipment of power. However, there is no problem found in the component of other LD10 material, which indicates that there is no problem in the ultimate voltage of $60-70 \mathrm{~V}$, as well as the boosting of the voltage in the process of oxidation, which is reasonable. Since the boosting speed of the LD10 material and the process of hard anodizing has no problem, so it is not a reason for the dissolution of the subcomponents.

\subsection{Analysis of the Unfair Distribution of Ampere Density}

As analyzed through the actual place of dissolved part, the film layer of the fixture hanging part is complete and there is no dissolution. If the fixture hanging part is not secure, it will cause too large inrush current that contacts point between parts and fixture and resultantly lots of heat will be generated. The high temperature of parts over the sulfuric acid surface accelerates the dissolving ability of the sulfuric acid, which makes the growth speed of the film surpass that of the hard anodizing, After the film layer to dissolve, the metal parts of the substrate will begin to dissolve. Thus, the damage of the parts will definitely occur at conductive place of the burning; however, being observed through the part of clamping of component, film layer's surface is complete without any trace of dissolution. Thus, we can get the conclusion that the statement that the loose condition between the components and clamping is the reason that causes current density to be uneven and components to be broken down, cannot hold water.

\subsection{Cause Analysis of Parts Dissolution Related to the Poor Radiating of the Parts' Partial Surface}

Because the component is of tubular shape, during the hard anodizing process the vertical racking makes the stirring air stay at the bottom of the bath, which will cause the cross sectional area of part to be too small, and the compressed air from bottom of the part cannot be released through the hole. Meanwhile, the part in the bath cannot be guaranteed to be absolutely vertical, which is likely to cause too high temperature of the center hole, the film dissolves faster than it grows, and the partial dissolution will occur. From the place and feature of the dissolution, it is easy to determine the main reason for the parts dissolving during the hard anodizing process is its poor radiation of the heat.

Studies show that the same parts in the previous production process undergo dissolution in the oxidation tank frequently, but the frequency and number of the parts are small. Compared with the previous production process, this part and its counterpart in the past basically share the same process parameters, solution components and fixture of the hard anodizing, the only relatively large change happened is the compressed air agitation lines which is laid at the bottom of existing oxidation tank.

According to the stress analysis of the bubbles in the liquid, when the compressed air stirs the solution, air bubbles will move from the bottle of the solution to the surface in a curved but not vertical way, which makes the compressed air uneven when it reaches the airline, and there is a big difference between the solution bubble center and the compressed air outlet position. This difference becomes larger and larger with the deepening of cross-sectional area of the solution, which cannot be accurately controlled, resulting in some difficulty in production.

During the actual parts operation, when the parts installing and hanging process is complete, they will enter into the hard anodizing tank. The operator usually firstly observes the solution agitation according to the size of the bubble on surface and chooses a good stirring position to locate the parts. However, due to the instability of the bubble movement, the liquid surface area of the bubble will change, which will affect the cooling effect of this portion of the part.

Observing from the position, the dissolution mainly appears in the middle and lower part of parts. This is mainly due to the $100 \mathrm{~mm}$ of the distance between the top of the part and the solution surface. Since the bubble continuously arrives to the solution surface and breaks over and over, the tremendous force generated by the bursting bubble plays a stirring action, so that the temperature of the shallow level of the liquid is even. The solution of the upper half part of the part continuously flows, the heat gets neutralized, which can maintain the continuous growth of the film, which is why the upper half of the part is not dissolved.

For hard anodizing bath with larger cross-sectional area and higher sides, the sole reliance on the stirring of the compressed air at the bottom of the bath just makes the solution within about $25 \mathrm{~mm}$ of the surface having a regular mixing, but the middle of the solution is poorly stirred, which is not conducive to the parts that have a small horizontal cross-sectional area. When the cross-sectional area of the part is large (such as a shell machine parts), the compressed air moves from the bottom upward and will be disturbed by the surface of parts, and the bubble changes the movement direction to improve the fluidity of the solution, which is one of reasons that the shell machine parts are not prone to be dissolved.

\subsection{The Measures Taken}

For the reasons discussed above, the key to solving such problems is to reduce the temperature of solution surface of the parts. Two main measures have been taken:

(1) To make improvement in the existing tooling as shown in Fig. (2). By changing the auxiliary cathode rod of the central hole of the parts into hollow tube, then add vent hole at the lower half of the tube wall of the hollow tube, and connect the hollow tube with a compressed air pipe to accelerate the cooling of the inner hole of the parts. Since the compressed air at the bottom of the tank rises unstably, the stirring function of compressed air cannot be simply relied on at the tank bottom.

For these parts, we should change the aluminum stick into hollow aluminum tube which is used during the oxidation process, and block the bottom of the aluminum tube, drill a small hole evenly by $1 / 3$ of scope near the bottom. As auxiliary cathode, it also plays the role of compressing the moving air. The aluminum stick is connected with the power cathode, and meanwhile, a plastic hose is used to get through the compressed air of the production line. Thus, the problem of stirring the solution in 
the inner hole of the parts during the process of hard anodizing is solved, which can be avoided by the parts dissolution.

(2) Improvement has been made in the arrangement of the stirring pipe in the hard anodizing tank, and a stirring pipe for the compressed air is also added, which improved the stirring ability of compressed air in the solution, as well as the inner hole heating of the parts. As for the ineffectiveness of solution's stirring after extending the existing tank crosssectional area, a manual pipe is added to the originally designed two-way compressed air production line to strengthen the solution stirring effect.

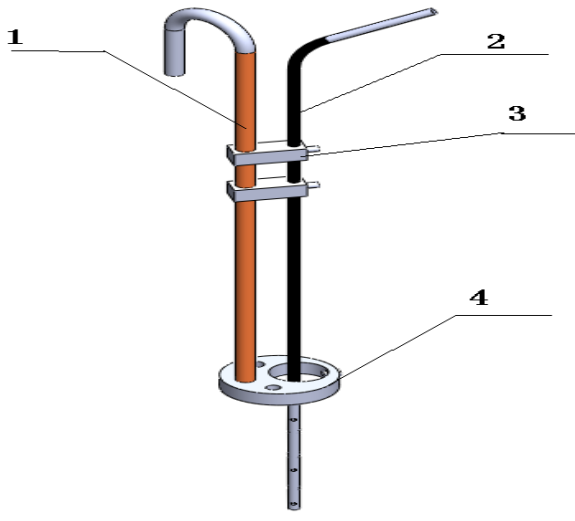

1 Anode-oxidation 2 Cathode-oxidation

3 Insulated Wood 4 Fixing Sleeve

Fig. (2). Schematic diagram of the fixture.

\subsection{Application Effect}

After the improvement of the parts of oxidation fixture, during the next few months of production, the phenomenon of dissolving in the tank could be effectively controlled, and there occurred no part dissolving. Meanwhile, after the successful implementation of the parts and application of this way to hard anodizing process of other tube parts, remarkable results have been achieved.

\section{CONCLUSION}

Through in-depth analysis of the dissolving phenomenon appearing during hard anodizing process of the aluminum alloy parts, this paper puts forward ideas and solutions, which has practical significance for solving the dissolution during the hard anodizing process.

\section{CONFLICT OF INTEREST}

The authors confirm that this article content has no conflict of interest.

\section{ACKNOWLEDGEMENTS}

This work is supported by the Natural Science Research Project of Shaanxi Provincial Education Department, China (No: 2013JK1122), the Education Reform Project of Xi'an International University, China (NO: 2013B47, 2013B18), the Planning Project of Shaanxi Provincial Science and Technology Department, China (NO: 2014JM8352).

\section{REFERENCES}

[1] G Yunxia, R Jijia, N Fuyuan. Aluminum Surface Treatment. Metallurgical Industry Press 2010; 286.

[2] Z Zufang. Aluminum Anodizing and Surface Treatment Technology. Chemical Industry Press 2004; 56-8.

[3] H Boyun, L Chenggong and S Likai. China Materials Engineering Canon Volume 4 Non-ferrous Materials Engineering (The First Volume), Chemical Industry Press 2006; 88-9.

[4] X Xuan, L Guohui, C Zichao. The Study on the factors that affect the aluminum anodizing film quality. Electrop Finish 2005; 24: 7-10.

[5] Zhou Y, L Bingen and S Zhisong. Research on aluminum room temperature hard anodizing technology. Materi Prote 2008; 41: 34 6.

[6] A Jiaju, W Xiaowei. Research on aluminum fast hard anodizing process under electric current. Electro Finish 2005; 24: 29-31.

[7] Z Liaoyuan, $Z$ Hong and $S$ Jianmin. Wide temperature fast aluminum material fast anodizing technology and application. Materi Prot 2001; 34: 37-9.

[8] W Dizhen. Research and application on aluminum exchange hard anodizing technology. Paint Electrop 2008; 23-6.

[9] A Miaozhong. Electroplating theory and technology. Harbin Institute of Technology Press, 2004; 280-1.

[10] Z Moyin. Aluminum room temperature hard anodizing technology mechanism of organic additives and bath liquid maintenance. Electroplating and Environmental Protection, 2002; 22: 28-30.

[11] L Youhou, J Yulan, H Ruoying. Hard anodizing of aluminum alloys with various electric wave forma. Electropl Finish 2001; 2: 16-9.

[12] S Jiwen, L Qimin. Aluminum and aluminum hard anodizing, J Sichuan Ordnance 2002; 32-5.

[13] W Sixiong. Aluminum hard anodizing failure analysis and prevention. Mater Prot 2003; 67-8.

Received: May 15, 2015

Revised: June 23, 2015

Accepted: July 4, 2015

(C) Peng and Li Licensee Bentham Open

This is an open access article licensed under the terms of the Creative Commons Attribution Non-Commercial License (http://creativecommons.org/licenses/ by-nc/4.0/) which permits unrestricted, non-commercial use, distribution and reproduction in any medium, provided the work is properly cited. 